\title{
Familial desminopathy: myopathy with accumulation of desmin-type intermediate filaments
}

\author{
Jiri Vajsar, Laurence E Becker, Robert M Freedom, E Gordon Murphy
}

\begin{abstract}
Two siblings developed cardiomyopathy several years before slowly progressive muscle weakness. Skeletal muscle biopsy specimens showed subsarcolemmal crescents of dark eosinophilic material in both type I and type II fibres. Immunohistochemically the subsarcolemmal material stained positively for the intermediate filament protein desmin and for the heat shock protein ubiquitin but for no other cytoskeletal proteins. Ultrastructurally the subsarcolemmal deposits consisted of aggregates of granular and filamentous material arising from $Z$ bands. Follow up muscle biopsies six years later showed an increased number of the muscle fibres that contained subsarcolemmal aggregates that stained positively for desmin and ubiquitin. These clinical and pathological features characterise a rare familial myopathy associated with an unusual distribution of desmin intermediate filament proteins in skeletal and probably also cardiac muscle.
\end{abstract}

$(\Im$ Neurol Neurosurg Psychiatry 1993;56:644-648)

Intermediate filaments represent one of the three main cytoskeletal protein filament systems of most vertebrate cells. The filaments, which are 10 to $15 \mathrm{~nm}$ wide, can be divided into four distinct sequence types, numbered I to IV. ${ }^{1}$ Acidic keratins are type I filaments; neutral-basic keratins, type II; desmin, vimentin, and glial fibrillary acidic protein, type III; and neurofilaments, type IV. Desmin and vimentin represent the main components of intermediate filaments in skeletal and smooth muscle cells. Immunoelectron microscopy has shown that desmin surrounds the myofibrils at Z-disc levels and anchors the myofibrils in the transverse register as well as to the sarcolemma. ${ }^{23}$ The network of intermediate filaments connects the plasma membrane skeleton with the nuclear lamina or karyoskeleton.

Abnormal expression of desmin has been seen in tumours derived from smooth and skeletal muscle, ${ }^{4}$ regenerating muscle fibres, ${ }^{5}$ and congenital and late onset myopathies with or without cardiomyopathy. ${ }^{6}$ Cardiomyopathy may develop during the course of the disease but has not been reported as a presenting feature.

We have previously reported on two sib- lings who presented with cardiomyopathy and clinically silent myopathy. ${ }^{7}$ During the following years, however, they have both developed slowly progressive muscle weakness. In this paper, we characterise histochemically, immunohistochemically, and ultrastructurally the increased number of deposits of subsarcolemmal material found in repeated muscle biopsy specimens.

\section{Patients and methods PATIENTS \\ Patient 1}

A five year old girl whose parents are second cousins presented with excessive tiredness, effort intolerance, and epigastric pain. Examination revealed gallop rhythm, enlarged left atrium, and deep T-wave inversion on ECG in the left precordial leads. Cardiac catheterisation at six years showed elevated left ventricular end diastolic pressure and pulmonary artery wedge pressure consistent with the reduced compliance of her left ventricle. There was also evidence of thickening of the left ventricular wall. Although she was not complaining of muscle weakness, an EMG performed at the age of 11 showed myopathic changes in the gastrocnemius muscle. Results of tests for nerve conduction, creatine kinase, lactate dehydrogenase, and concentrations of total and free carnitine in serum were all normal. She then had an open muscle biopsy from the triceps muscle. By the age of 17 she had developed muscle weakness. Examination showed generally reduced muscle bulk and diminished deep tendon reflexes. Muscle strength was decreased to four fifths in her arms and legs; proximal muscle groups were slightly weaker. She had an incomplete Gowers' sign and tired easily when running. The results of her mental examination were normal. Electrocardiographic examination showed left ventricular hypertrophy with strain. The results of repeated measurements of creatine kinase in serum and of forearm lactic ischaemic tests were normal. A second biopsy of the triceps muscle was performed.

\section{Patient 2}

The first patient's brother developed transient congestive heart failure at two years. Examination revealed palpable gallop and quadruple rhythm with third and fourth heart sounds. ECG showed decreased contractility of the left ventricle and diffuse S-T depression and T-wave inversion in the left precor- 
Figure 1 Muscle biopsy specimen showing subsarcolemmal deposits of dark eosinophilic material in most muscle fibres (HE, $\times$ 800).

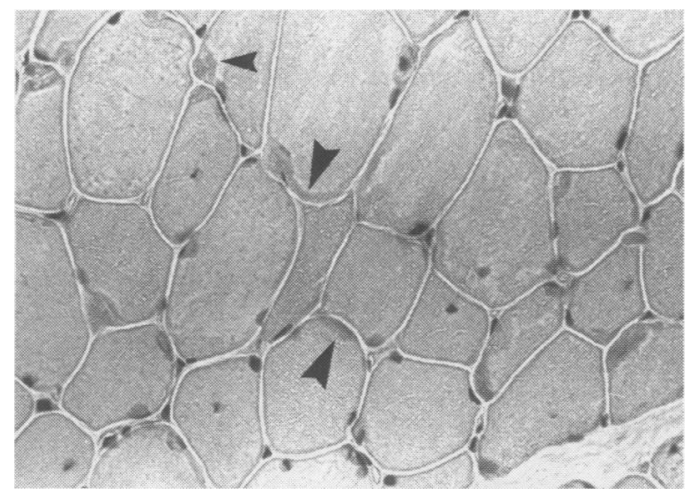

dial leads. This boy, born at term, had developed hypoxic-ischaemic encephalopathy because of meconium aspiration at birth. This was followed by a seizure disorder and global developmental delay: he walked at the age of $31 / 2$ years and spoke only single words at the age of 6 . Electromyography yielded normal results and his serum creatine kinase concentration was normal at the age of 5 when he underwent a biopsy of the triceps muscle. By the age of 11 he had difficulty rising from the floor. Examination indicated he was mentally retarded. Muscle bulk was reduced and muscle strength was four fifths diffusely but slightly better distally. Deep tendon reflexes were brisk and a partial Gowers' sign was present. Electrocardiographic examination showed left ventricular hypertrophy with strain. Serum creatine kinase concentration was again normal and another biopsy of the triceps muscle was performed.

\section{MUSCLE BIOPSIES}

Two open biopsies from the right triceps muscle for each child were performed 6 years apart. Serial sections embedded in paraffin and frozen were prepared for conventional histological, histochemical, and immunohistochemical examinations (table). Dewaxed and rehydrated paraffin sections were stained with commercially available antibodies (Dakopatts, Denmark) to localise desmin and ubiquitin immunoreactivity. Semi-thin section fixed with osmium and stained with toluidine blue were examined and thin sections

Table Results of staining subsarcolemmal deposits in all four biopsy specimens in two siblings with cardiomyopathy and progresssive muscle weakness

\begin{tabular}{ll}
\hline Stain & Staining or reactivity \\
\hline Gomori's modified trichrome & Dark green \\
Hematoxylin and eosin & Dark eosinophillic \\
Pyronin & Negative \\
Periodic acid-Schiff & Positive \\
Phosphorylase & Negative \\
Acid phosphatase & Positive \\
Menadione nitroblue tetrazolium & Negative \\
Succinic dehydrogen & Negative \\
Lactic dehydrogenase & Negative \\
Oil red O & Negative \\
Congo red & Negative \\
Cytochrome oxidase & Negative \\
Desmin & Dark brown \\
Myosin & Negative \\
$\alpha$ Actinin & Negative \\
Actin & Negative \\
Vimentin & Negative \\
Ubiquitin & Positive \\
\hline
\end{tabular}

obtained for electron microscopy.

Limited muscle biochemistry showed normal activity of the following glycolytic enzymes: acid maltase, debranching enzyme, phosphorylase, phosphofructokinase, phosphoglycerate kinase, and phosphoglycerate mutase.

\section{PATHOLOGICAL RESULTS}

Light microscopy, histochemistry, and immunohistochemistry

Mild variation in fibre size and a few atrophic fibres were seen on cross and longitudinal sections in both patients. No fibre degeneration or regeneration, however, was present in any of the four specimens. There was increased internalisation of nuclei and mild focal endomysial fibrosis. The most striking finding was the presence of deposits of dark eosinophilic subsarcolemmal material (fig 1). The subsarcolemmal deposits showed identical positive staining in all four specimens (table). On ATPase, the material was seen in both type I and II fibres.

The histogram showed that $75 \%$ (patient 1) and $70 \%$ (patient 2) of fibres were type $I$. The average diameter of type I fibres in patient 1 increased over six years from 32 to $45 \mu$ and the average diameter of type II fibres from 43 to $56 \mu$. In patient 2 the size of type I fibres increased over six years from 21 to $36 \mu$, whereas the size of type II fibres decreased from 25 to $21 \mu$.

When the immunoperoxidase technique with antisera to myosin, ubiquitin, vimentin, desmin, $a$ actinin, actin, keratin, and glial fibrillary acidic protein was performed, crescents of subsarcolemmal material stained positive only with antisera to desmin (fig $2 a, 2 b$ ) and ubiquitin (fig 3 ). The muscle fibres containing material positive for desmin were calculated in three different microscopic fields (50 fibres/field). In patient 1 the deposits were initially present in an average of $22 \%$ of muscle fibres, which increased to $68 \%$ in the second biopsy from the same muscle six years later. In patient 2 the material was present initially in $14 \%$ and later in $66 \%$ of muscle fibres.

\section{ELECTRON MICROSCOPY}

The findings on electron microscopy were similar for both children. There were focal subsarcolemmal areas of muscle fibres that showed aggregates of granular and filamentous material (fig 4, fig 5a, 5b). The aggregates varied in size and occasionally could be discerned arising and streaming from $\mathrm{Z}$ bands. Where the material was densely packed filaments could not be distinguished. Areas of extensive aggregates showed mild accumulation of glycogen. Both the I-bands and Z-bands were distorted in areas adjacent to the electron-dense aggregates. Otherwise the myofibrillary alignment was normal. In addition, there was extensive accumulation of mitochondria in the sarcoplasm beneath the electron dense aggregates. The mitochondria were mildly pleomorphic; some were elongated or branched. Contiguous to the subsar- 

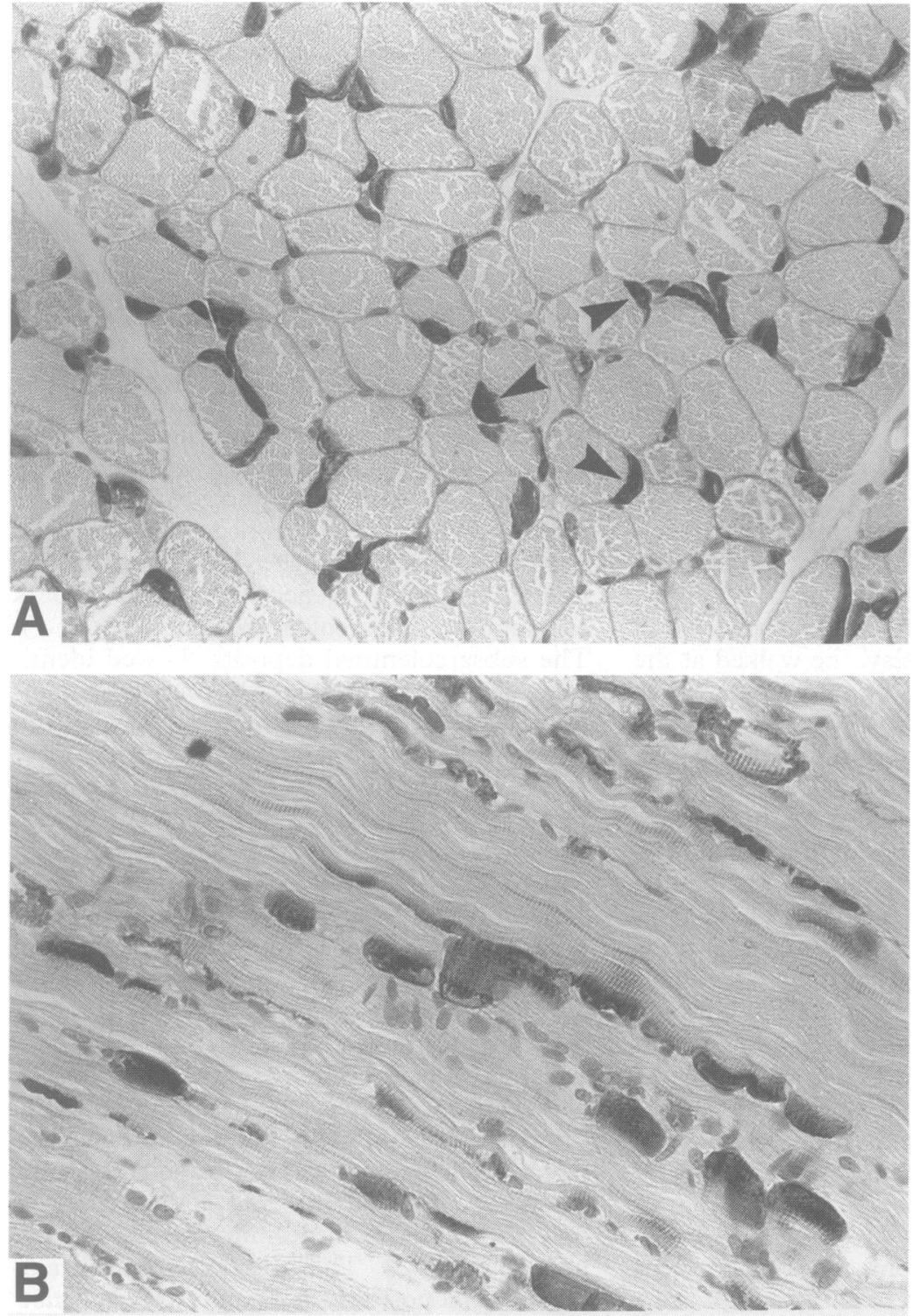

Figure 2 Sections of triceps muscle of patient 2 showing subsarcolemmal crescents of deposits of desmin-positive material with immunoperoxidase stain. (a) Transverse section $\times 320$, (b) longitudinal $\times 400$.

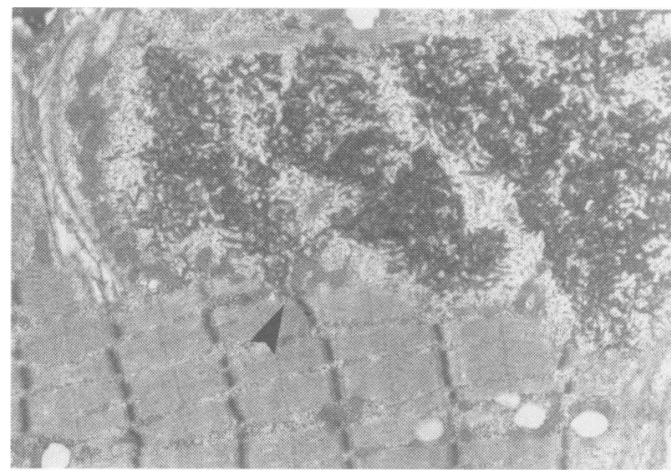

Figure 4 Electron micrograph of triceps muscle showing subsarcolemmal deposit in which aggregates seem to be arising and streaming from $Z$-bands ( $\times 11600)$.

\section{Discussion}

We have described the unique clinical and pathological findings in two siblings who presented with cardiomyopathy several years before they developed slowly progressive muscle weakness. Although initially there was no clinical evidence of skeletal myopathy, biopsy specimens from the triceps muscles showed crescents of subsarcolemmal material in $22 \%$ of muscle fibres in patient 1 and in $14 \%$ in patient 2 ; six years later, when muscle weakness had developed, this material increased to $68 \%$ and $66 \%$, respectively. The subsarcolemmal material in all four specimens stained positively for the intermediate filament protein desmin and the heat shock protein ubiquitin. Electron microscopy showed that the subsarcolemmal crescents contained aggregates of granular and filamentous material. The aggregates streamed from Z-bands.

Patients with non-progressive or slightly progressive myopathies may have various morphological changes in the muscle fibres. In some myopathies the morphological abnormality may be characterised by an accumulation of abnormal material seen in various parts of the cytoplasm with both light and electron microscopy. ${ }^{70}$ The material is often electron dense at the ultrastructural level and consists of granules and intermediate filaments approximately $10 \mathrm{~nm}$ node. In the 15 case reports presented by Pellissier et al the electron dense material was immunoreactive for desmin and in one case the material was closely related to Z-bands. ${ }^{6}$ In our two patients the material positive for desmin was found in subsarcolemmal areas and also appeared related to Z-bands.

Most of the cases reported by Pellissier et $a l$, as well as ours, were familial, with age of onset between 2 and 48 years. $^{6}$ All three of their cases involving children and some of their adult patients developed cardiomyopathy after the onset of muscle weakness. Two children, aged 11 and 13 years, died of heart failure. ${ }^{10}$ On the other hand, our patients presented with cardiac symptoms early in life, long before their symptoms of muscle weakness occurred. They predominantly experienced proximal muscle weakness that was slowly progressive over the years.
Figure 3 Subsarcolemmal deposits showing positive stain, $(\times 1000)$ 

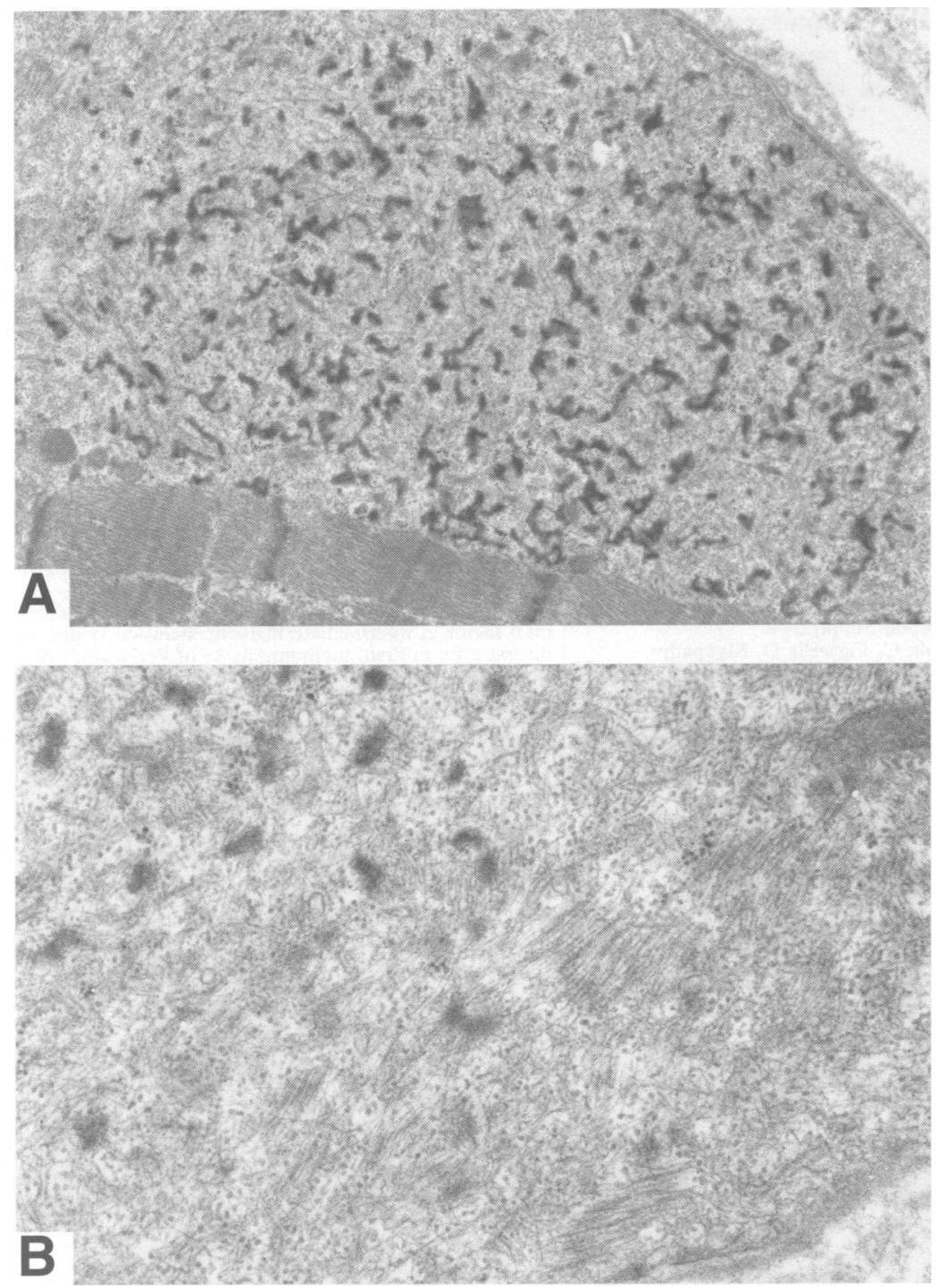

Figure 5 (a) Low magnification electron micrograph showing aggregates of subsarcolemmal granular and filamentous material $(\times 18$ 870). (b) High magnification of abnormal material showing mainly filaments and few granules ( $\times 68000)$.

Most patients reported also have slowly progressive muscle weakness, mostly proximal, but few have diffuse weakness with predominantly distal involvement. ${ }^{11}$ In our patients the progressive nature of the myopathy was supported by the findings of repeat biopsies. In the second biopsy from the same muscle, there were more fibres displaying accumulation of material positive for desmin in a characteristic subsarcolemmal distribution.

Desmin, a polypeptide consisting of nonhelical $\mathrm{N}$-terminal and C-terminal ends and an $\alpha$ helical middle domain, has a molecular weight of $52000 \mathrm{~d}$ and consists of about 300 amino acids. Monoclonal or polyclonal antibodies against desmin may have specificity against any portion of the desmin molecule, which has about $30 \%$ to $70 \%$ of amino acids in common with other intermediate filaments. ${ }^{4}$ During early development the primary generation of myotubes expresses both desmin and vimentin. Both are also expressed in the early stages of activated satellite cells seen in regenerating muscle. ${ }^{2512}$ With maturation in both myotubes and satellite cells, vimentin is lost, whereas desmin persists.
Sarnat found very strong immunoreactivity for desmin and vimentin in the myofibres of neonates with myotubular myopathy. ${ }^{12} \mathrm{He}$ suggested that the high level of expression of intermediate filament proteins suggests the arrest of morphogenesis in the developing muscle fibres. In some myopathies and in the strained muscle of elderly people, increased stainability for desmin in areas of Z-disc streaming also occurs, suggesting that intermediate filaments are related to sarcomerogenesis or repair of disrupted sarcomeres. ${ }^{213}$

During normal myogenesis in avian and mammalian muscle, desmin intermediate filament proteins organise from a longitudinal to a transverse circumferential network around Z-discs. When Tassin et al treated normal mice myotubes with tetrodotoxin, which prevents contractile activity of myotubes by blocking the voltage-dependent sodium ion channels, the treated myotubes displayed aggregates of desmin intermediate filament protein. ${ }^{14}$ Also the normal organisation of desmin from a longitudinal to a transverse network was prevented. Both of these results were reversible: when the treatment with tetrodotoxin was stopped the desmin aggregates disappeared and the normal organisation of the desmin network followed. These results suggested that aggregates may reflect a modification of binding desmin with the plasma membrane in the presence of an abnormal agent.

Abnormal reorganisation of desmin intermediate filaments caused by an unknown deleterious process is supported by another finding. A variety of cellular stresses including viral infection, toxicological injury, and experimental heat shock have been suggested as the cause of collapse of intermediate filaments with ubiquitination in neurons, astrocytes, and liver and muscle cells. ${ }^{15}$ The ubiquitin and desmin-positive aggregates in our patients may thus represent a reorganised and collapsed network of desmin intermediate filaments.

Thus increased immunoreactivity to desmin seems to be a non-specific feature indicating perturbation in both the developing and mature muscle fibres. The cause of the progressive accumulation of aggregates positive for desmin, however, seen in muscle fibres of our patients and patients reported elsewhere, remains unclear. In addition, it is not clear why the heart muscle in our patients was initially affected, a feature rather different from the other reported cases of myopathies with desmin accumulation. Progressive disorganisation of desmin intermediate filaments in mature muscle fibres in response to deleterious stimuli with higher affinity for the cardiac muscle is one possibility. Another may be the existence of a genetic predisposition for excessive production or incorrect turnover of desmin. A recent study localised the gene encoding the synthesis of desmin on chromosome $2 \mathrm{q} 35,{ }^{16}$ but so far the isolated desmin probe has not been used in clinical practice. Finally, the fundamental issue of whether primary desmin accumulation may result in a 
myopathy or whether it is a secondary result of progressive changes in the muscle caused by some other undetermined factor needs to be resolved.

This manuscript was prepared with the assistance of medical publications, The Hospital for Sick Children, Toronto, Ontario.

1 Steinert PM, Roop DR. Molecular and cellular biology of intermediate filaments. Annual Review Biochemistry 1988;57:593-625.

2 Thornell L-E. Immunohistochemistry of intermediate filaments [abstract]. ₹ Neurol Sci 1990;98 (Suppl):58.

3 Granger BL, Lazarides $\mathbf{E}$. The existence of an insoluble $Z$ disc scaffold in chicken skeletal muscle. Cell 1978, disc scaffold

4 Truong LD, Rangdaeng S, Cagle P, Ro JY, Hawkins $H$ Font RL. The diagnostic utility of desmin. A study of 584 cases and review of the literature. Am $\mathcal{F}$ Clin Patho 1990;93:305-14.

5 Bornemann A, Schmalbruch $\mathrm{H}$. The intracellular distribution of desmin and vimentin in regenerating rat muscles [abstract]. I Neurol Sci 1990;98:(Suppl):294.

6 Pellissier JF, Pouget J, Charpin C, Figarella D. Myopathy associated with desmin type intermediate filaments. An immunoelectron microscopic study. $尹$ Neurol Sci 1989 ; 89:49-61.

7 Calderon A, Becker LE, Murphy GE. Subsarcolemmal vermiform deposits in skeletal muscle, associated with familial cardiomyopathy: report of two cases of a new entity. Pediatr Neurosci 1987;13:108-12.

8 Shafiq SA, Sande MA, Carruthers RR, Killip T, Milhorat
AT. Skeletal muscle in idiopathic cardiomyopathy. $f$ Neurol Sci 1972;15:303-20.

9 Fardeau M, Godet-Guillain J, Tome FMS, et al. Une nouvelle affection musculaire familiale, définie par l'accumulation intra-sarco-plasmique d'un matériel granulo-filamentaire dense en microscopie électronique. Rev Neurol (Paris) 1978;134:411-25.

10 Goebel HH, Lenard HG, Langenbeck U, Mehl B. A form of congenital muscular dystrophy. Brain Dev 1980;2. $387-400$.

11 Edström L, Thornell L-E, Eriksson A. A new type of hereditary distal myopathy with characteristic sarcoplasmic bodies and intermediate (skeletin) filaments. $\mathcal{F}$ Neurol Sci 1980;47:171-90.

12 Sarnat HB. Myotubular myopathy: arrest of morphogenesis of myofibres associated with persistence of fetal sis of myofibres associated with persistence of fetal vimentin and desmin. Four cases compared with fetal

13 Jakobsson F, Borg K, Edström L. Fibre-type composition, Jakobsson F, Borg K, Edström L. Fibre-type composition,
structure and cytoskeletal protein location of fibres in anterior tibial muscle. Comparison between young adults and physically active aged humans. Acto Neuropathol (Berl) 1990;80:459-68

14 Tassin AM, Pincon-Raymond M, Paulin D, Rieger F. Unusual organization of desmin intermediate filaments in muscular dysgenesis and TTX-treated myotubes. Dev Biol 1988;129:37-47.

15 Lowe J, Blanchard A, Morrell $\mathrm{K}$, et al. Ubiquitin is a common factor in intermediate filament inclusion bodies of diverse type in man, including those of Parkinson's disease, Pick's disease, and Alzheimer's disease, as well as ease, Pick's disease, and Alzheimer's disease, as well as Rosenthal fibres in cerebellar astrocytoma, cytoplasmic bodies in muscle, and Mallory

16 Viegas-Pequignot E, Li ZL, Dutrillaux B, Apiou F, Paulin $D$. Assignment of human desmin gene to band $2 \mathrm{q} 35$ by nonradioactive in situ hybridization. Hum Genet 1989; 83:33-6. 